\title{
Influencia del advergaming en los jóvenes universitarios de Lima
}

\author{
Recibido: 20 de enero de 2014 \\ Aceptado: 30 de julio de 2014 \\ Publicado: 28 de noviembre de 2014
}

\author{
Alice Garrido Gallardo \\ agarrido@globokas.com
}

Universidad de San Martín de Porres (Perú)

\begin{abstract}
Resumen: En tiempos donde el consumidor es capaz de encontrar lo que desea sin ser interrumpido por la publicidad, los anunciantes ven necesaria la tarea de expandir sus mensajes por medio de nuevas alternativas que acerquen al público con la marca de manera atractiva. El advergame forma parte de estas nuevas herramientas ya que busca fidelizar al consumidor mediante juegos interactivos que representen los valores, características y beneficios de la marca. Para conocer cuál es la influencia de esta herramienta publicitaria en los jóvenes de la ciudad de Lima, este artículo realiza un análisis sobre la actitud que ellos generan hacia la marca dependiendo del tratamiento que se le da en el juego, el tiempo de exposición y otras variables.
\end{abstract}

Palabras clave: Publicidad, posicionamiento, advergaming, advertainment, juegos, influencia, actitud hacia la marca.

\begin{abstract}
In times where the consumer is able to find what he wants without being interrupted by advertising, advertisers are required to expand their messages through new alternatives that connect the audience with the brand in an attractive way. The advergame is part of these new tools as it seeks to retain consumers through interactive games that represent the brand's values, characteristics and benefits. To understand the influence of this advertising tool in Lima's youth, this article makes an analysis on the attitude they generate towards the brand depending on the treatment that is given in the game, the exposure time and other variables.
\end{abstract}

Key words: Advertising, Positioning, Advergaming, Advertainment, Games, Influence, Attitude towards the brand. 


\section{Introducción}

Los anunciantes se encuentran en una constante búsqueda de nuevas maneras efectivas de transmitir mensajes a sus posibles consumidores. La saturación de los medios publicitarios tradicionales, sus altos costos, la introducción de contenido "chatarra" y sus consecuencias en los consumidores como es el zapping ${ }^{1}$ o la simple omisión de los mensajes han llevado a los publicistas a buscar nuevas maneras de alcanzar a sus público objetivo.

Por ello, no ha de sorprender que gracias al avance tecnológico y a la masificación de medios alternativos, nuevas herramientas de mercadotecnia como el advertainment ${ }^{2}$ haya provisto a los anunciantes de una nueva alternativa para dirigirse a sus audiencias.

Se puede decir que en la actualidad los jóvenes son capaces de ser fieles al contenido que ofrecen los medios pero no necesariamente a los medios mismos y en mucho menor grado a la publicidad que se transmite en ellos, sobre todo en televisión donde el zapping es la amenaza número uno de los anunciantes. Bringué y Sádaba (2007) manifestaron que "los jóvenes latinoamericanos prefieren Internet por sobre la televisión, incluso aunque no dispongan de acceso a la red en su hogar". Asimismo, revelaron que "el tiempo libre que pasa un adolescente buscando entretenimiento en la web es significativamente mayor al que le dedica a los medios tradicionales".

En este sentido los anunciantes se han visto forzados a renovar sus esfuerzos publicitarios en la web y en distintos soportes que sean capaces de fusionar a su marca con el entretenimiento que busca este target. Es así que gracias al crecimiento conjunto de la industria de los videojuegos y el número de usuarios que navegan en la web en busca de juegos online, herramientas como el advergaming, la práctica de usar videojuegos para publicitar una marca, producto, organización o idea ( $c f$. Pons, 2006a), un derivado del advertainment, se posiciona como una de las mejores opciones en la actualidad para captar al consumidor de manera atrayente ya que no crea conflictos con su objetivo de entretenerse sino que se basa justamente en ese concepto para acercar a la marca con el público.

El advergaming es la creación de videojuegos que no solo muestran a la marca en el transcurso del mismo, sino que todo el entorno del juego se ve relacionado con los valores de la misma, de manera que el usuario interactúa con ella y aprende de su entorno. Mediante un juego es más fácil asimilar el mensaje. El usuario está aprendiendo, informándose o conociendo algo nuevo, mientras se está divirtiendo.

\footnotetext{
1 De acuerdo con el Diccionario panhispánico de dudas (2005), el “zapeo" es "acción de cambiar reiteradamente de canal de televisión por medio del mando a distancia".

2 Unión de dos términos: Advertising ("publicidad") y Entertainment ("entretenimiento").
} 


\section{Objetivos}

\subsection{Objetivo general}

Conocer la influencia publicitaria del advergaming y verificar si ocasiona cambios de actitud hacia la marca promovida en los jóvenes de la Universidad de Lima.

\subsection{Objetivos específicos}

- Identificar si la frecuencia de uso y tiempo de exposición a los juegos influencian en la actitud hacia la marca por parte del target.

- Analizar qué tratamiento se le debe dar a la marca en los juegos para provocar y cambio en la actitud del público joven limeño.

- Identificar qué tipo de productos o servicios pueden influenciar con mayor facilidad con esta herramienta publicitaria.

\section{Metodología}

Se requiere la realización de técnicas de recolección de datos cuantitativas para lograr información significativa de la población a estudiar. Estas técnicas nos permiten alcanzar un número mayor de sujetos de estudio. De esta manera se podrá concluir si el objeto de estudio es, en efecto, una estrategia que se adecúa a los requerimientos de los usuarios.

\subsection{Población y muestra}

\subsubsection{Población}

Según el ranking de America Economía (2013), la cantidad de alumnos de pregrado de la Universidad de Lima rondó los 14,109 estudiantes en el año 2012.

\subsubsection{Muestra}

Para nuestra investigación se contó con una muestra de 137 consumidores.

\subsection{Operacionalización de las variables}

\subsubsection{Variable dependiente}

El objetivo principal del trabajo se alcanzará mediante la evaluación de la siguiente variable dependiente: actitud hacia la marca. 
Figura 1: Medición de variable dependiente.

\begin{tabular}{|c|c|c|c|}
\hline $\begin{array}{c}\text { VARIABLE } \\
\text { DEPENDIENTE }\end{array}$ & INDICADORES & CATEGORÍAS & ESCALA \\
\hline Actitud hacia la marca & Reacciones luego de haber jugado & $\begin{array}{l}\text { - } \text { Consumo } \\
\text { - Identificación } \\
\text { - Recordación } \\
\text { - No hay cambios }\end{array}$ & Ordinal \\
\hline
\end{tabular}

Fuente: Elaboración propia.

\subsubsection{Variables independientes}

Las que se obtendrán a través de la cuantificación y combinación de las siguientes variables independientes:

- $\quad$ Tratamiento de la marca en el juego.

- $\quad$ Tiempo de exposición al juego.

- $\quad$ Frecuencia de uso del juego.

- Plataforma.

- Sexo.

Figura 2: Medición de variables independientes.

\begin{tabular}{|c|c|c|c|}
\hline $\begin{array}{c}\text { VARIABLES } \\
\text { INDEPENDIENTES }\end{array}$ & INDICADORES & CATEGORÍAS & ESCALA \\
\hline Tratamiento de marca & $\begin{array}{l}\text { Características de la marca } \\
\text { integradas al juego }\end{array}$ & $\begin{array}{ll}\text { - } & \text { Personaje } \\
\text { - } & \text { Premio } \\
\text { - Escenario } \\
\text { - Entorno }\end{array}$ & Nominal \\
\hline Exposición & $\begin{array}{l}\text { Horas expuestas en cada } \\
\text { sesión de juego }\end{array}$ & $\begin{array}{l}\text { - } \quad \text { Más de } 3 \text { horas } \\
\text { - } 2 \text { horas } \\
\text { - } 1 \text { hora } \\
\text { - } \quad \text { Menos de } 1 \text { hora }\end{array}$ & Ordinal \\
\hline Uso & $\begin{array}{l}\text { Cantidad de días por semana } \\
\text { dedicadas al juego }\end{array}$ & $\begin{array}{ll}\text { - } & \text { Todos los días } \\
\text { - } & 3 \text { veces por semana } \\
\text { - } & 1 \text { vez por semana } \\
\text { - } & \text { Esporádicamente }\end{array}$ & Ordinal \\
\hline Sexo & Caracteres sexuales & $\begin{array}{l}\text { - Masculino } \\
\text { - Femenino }\end{array}$ & Nominal \\
\hline Producto o servicio & Tipo de producto inmerso en el juego & $\begin{array}{l}\text { - } \text { Bebidas gaseosas } \\
\text { - Fast foods } \\
\text { - Ropa } \\
\text { - Snacks } \\
\text { - Otros }\end{array}$ & Nominal \\
\hline
\end{tabular}

Fuente: Elaboración propia. 
Estas se evaluarán a través de la frecuencia relativa o los porcentajes que tengan las diferentes categorías de cada una de las variables en estudio.

\subsection{Técnicas de recolección de datos}

La encuesta comprende un conjunto de preguntas seleccionadas en un cuestionario, para conocer la opinión del público sobre la investigación que se está realizando. La cantidad de participantes fue de 137 sujetos. El instrumento que se utilizó fue el cuestionario, elaborado con preguntas cerradas y opciones múltiples.

\subsection{Técnicas para el procesamiento de la información}

- Agrupación y clasificación de datos en tablas de frecuencia.

- Gráficos y tablas que permitan visualizar la información.

- Medidas de tendencia central como la moda y los percentiles.

- Medidas de dispersión de ser necesario.

\subsection{Aspectos éticos}

Por tratarse de una población joven en el que algunos participantes no alcanzan la mayoría de edad no se consideraran ejemplos ni ningún material publicitario que hagan alusión directa o indirecta a los siguientes contenidos: bebidas alcohólicas, tabaco, drogas, sexo, discriminación de cualquier tipo y violencia.

\section{Hipótesis de trabajo}

\subsection{Hipótesis principal}

El advergaming es una de las herramientas publicitarias mejor calificadas por el target estudiado en contraste con los medios tradicionales, y que ocasiona un alto porcentaje de cambio en actitud hacia la marca en los mismos.

\subsection{Hipótesis específicas}

- La frecuencia de uso y tiempo de exposición a los advergames influyen de manera positiva con el cambio en la actitud hacia la marca.

- Los advergames que generan mayor influencia en el target son aquellos en los que todo el entorno está asociado con la marca. 
- Los productos que son más fáciles de vincular con los advergames son los snacks y bebidas gaseosas.

\section{Publicidad en los videojuegos}

Para Mencía (2012), “los estudios más recientes demuestran que las aplicaciones móviles más utilizadas por los usuarios de smartphones son los juegos [...] más del $60 \%$ de los usuarios juegan con su móvil con una media mensual de casi 15 horas, un número de horas interesante y más si asumimos que jugando es como mejor asimilamos conceptos, conocimientos y experiencias". Agrega el mencionado autor:

"Con la publicidad tradicional, el consumidor tiene un contacto puntual con la marca, de no más de 30 segundos en un anuncio en televisión o mucho menos tiempo si hablamos de publicidad en prensa. Con un videojuego corporativo, ese contacto entre marca y consumidor puede extenderse durante días, semanas e incluso meses.

Además, el engagement del usuario con la marca es mucho mayor, ya que este último participa en el mensaje, en el propio contenido que la empresa ha diseñado como guión del juego. Para que la fidelización del consumidor sea efectiva, es necesario analizar qué percepción tiene el cliente de la empresa, de modo que seamos capaces de desarrollar como marca una historia dentro del videojuego consistente e impactante, que transmita fielmente los valores de la empresa y su utilidad para el usuario".

Carcelén y Sebastián (2010) explican que los videojuegos se han convertido "en una de las plataformas más utilizadas por los anunciantes que buscan nuevas fórmulas para hacer llegar sus mensajes de forma eficaz, algo que resulta lógico teniendo en cuenta los índices de efectividad que reflejan algunos estudios. Las nuevas técnicas publicitarias experimentan una mayor aceptación entre los usuarios; un público cansado de la falta de originalidad de los viejos soportes, y que demanda una mayor personalización de los mensajes”. Asimismo, explica:

"La capacidad de interacción de los videojuegos han resultado clave de la nueva tendencia, y los resultados avalan su éxito: según Nielsen, en el año 2008 hasta un $87 \%$ de los jugadores ${ }^{3}$ recordó las marcas aparecidas en los videojuegos a través del Advergaming".

\footnotetext{
3. Un cambio considerable es la ampliación del target tradicional. Lejos de lo que se podría suponer, son más las personas mayores de 36 años las que juegan videojuegos con un $41.6 \%$ a comparación de los adolescentes con un $29.7 \%$. Asimismo, cada vez son más las mujeres que practican estos juegos, disminuyendo la brecha a tan solo un $58.1 \%$ para los hombres contra un $41.9 \%$ para las mujeres.
} 
Pons (2006b) enumera las ventajas principales de esta herramienta:

- "Publicidad no molesta: Introducida dentro del contenido que estás disfrutando.

- Interactividad: Nos permite conocer cuáles son los gustos del cliente a través de juegos sencillos, que a la vez puedan propiciar una mayor implicación con el mensaje e identificación con la marca. Mientras que una campaña de publicidad eleva un $2 \%$ la intención de compra o aceptación del producto en el público expuesto, la combinación de esta con el marketing online incrementa este índice hasta $12 \%$, según la Asociación de Publicidad Interactiva Europea.

- Personalización: Al ser interactiva y poder conocer la persona tras el anuncio, es posible ajustar la publicidad a cada persona y momento del día.

- Eficacia: Los elevados tiempos de contacto entre la marca y el cliente son imposibles con un pop up cuya eficacia no es mayor a 2 segundos, o en los 30 segundos que pueda durar un anuncio en televisión. Sin embargo, un consumidor puede disfrutar de 5 a 35 minutos, de promedio, con un juego publicitario.

- Marketing viral: Este dato me sorprendió, para ser sinceros. Un estudio realizado recientemente con Orbitz, señala que la funcionalidad 'tell a fried' (envío a un amigo) para recomendaciones de conocidos, creó 1.8 millones de envíos, lo que supera y refuerza el conocimiento de una marca a través del 'boca a boca', mientras que la exposición del público, únicamente a una campaña de publicidad en televisión, genera un índice de recuerdo del 14\%. La combinación de televisión y medios online permite incrementar este índice hasta un 44\%”.

Marti Parreño (2005) añade otras ventajas del uso del advergame en campañas publicitarias:

- "Máximo grado de atención por el usuario: Cuando una persona juega pone todos sus sentidos dentro del juego por lo que son capaces de recepcionar al instante cualquier nuevo elemento que aparezca durante la trama del mismo. Frente a cualquier otro medio, la aparición de un elemento nuevo requerirá de toda la atención por parte del usuario ya acostumbrado a no dejar pasar ninguna pista u obstáculo para poder ganar el juego.

- Mayor tiempo de retención de usuario: A diferencia de cualquier otro medio publicitario tradicional, el advergame puede mantener 
al usuario centrado en él durante una buena cantidad de tiempo, siendo la media para las campañas de éxito más reconocidas como Coca-Cola o Burger King de al menos 20 minutos por sesión de juego. Dándole tiempo de sobre al anunciante para poder incrustar en la trama valores asociados a la marca.

- No se agota: El advergame posibilita algo que ningún otro advergame puede lograr. El usuario puede volver a él más de una vez, incluso en el mismo día. No existe otro recurso que permita este tipo de interacción y re búsqueda del material publicitario".

Medina (2007) manifiesta que "en cuanto al advergaming, la práctica de emplear los videojuegos para fundir en ellos el mensaje publicitario, es un fenómeno que no para de crecer. Prácticamente en todos los hogares de Estados Unidos hay ya una videoconsola, y Europa le sigue los pasos muy de cerca. Ese enorme potencial de videojugadores americanos, hace que la cifra de publicidad en los videojuegos se acerque ya a los 500 millones de dólares, una cifra que tiende a crecer de manera vertiginosa". Hace acotación a lo siguiente:

"Hasta hace muy poco tiempo eran los productores de los videojuegos los que pagaban importantes royalties a las marcas, para que estas les permitiesen usar sus logotipos en los juegos, de cara a dotar a estos de un mayor realismo. Sin embargo, ahora es Pizza Hut quien le paga a Sega para que los Crazy Taxis usen como parada habitual uno de sus establecimientos; o marcas como Nike y Coca-Cola pagan a Playstation para incluir vallas de sus productos en las calles de las ciudades virtuales de sus juegos.

Advertainment, Advergaming, Artvertising, Tryvertising y muchas otras formas nuevas de publicidad, están irrumpiendo con fuerza en el mercado. Rompiendo los viejos esquemas, acabando con las viejas rutinas del sota, caballo y rey de los medios convencionales, y abriendo ante nosotros un nuevo panorama, lleno de sugerentes perspectivas".

\section{Resultados}

\subsection{Frecuencia y uso de los videojuegos}

De los 137 alumnos encuestados, el 45\% asegura practicar juegos de video al menos 3 veces por semana. Esto quiere decir que la mayoría de jóvenes de la Universidad de Lima se exponen a este medio con una periodicidad considerable como para tomarla en cuenta como herramienta publicitaria. Sin embargo, un $37 \%$ asegura jugar únicamente de vez en cuando.

Asimismo, el 58\% de los encuestados aseguran que le dedican más de 2 horas a cada sesión de juego. Esto quiere decir que los juegos cuentan con un tiempo de exposición 
continuo muy elevado, lo cual permite introducir mensajes que se transmitirán de manera ininterrumpida por un largo periodo.

\subsection{Tipo de producto o servicio que se asocia más con el advergame}

De los 137 alumnos encuestados, el 32\% considera que el rubro que mejor se asocia a los advergames es el de bebidas gaseosas, seguido por un $26 \%$ que considera a los snacks como los productos que mejor se relacionan con esta herramienta. Esto indica que a pesar de no haber experiencias previas con advergames de este rubro, el target lo considera como uno de los mejores productos que se adecúan a esta herramienta.

\subsection{Mejor herramienta publicitaria}

El $43 \%$ de los encuestados opina que el mejor medio para hacer publicidad actualmente es la Internet, seguido del advergame (25\%), un porcentaje más alto incluso que el de la propia televisión (20\%). Esto quiere decir que el target estudiado rechaza la publicidad tradicional (mayormente intrusiva) y que prefiere buscar la conexión con la marca. Muy por detrás aparece la radio con un $9 \%$ de preferencia y la prensa escrita con un 3\% (figura 3 ):

Figura 3: Mejor herramienta publicitaria.

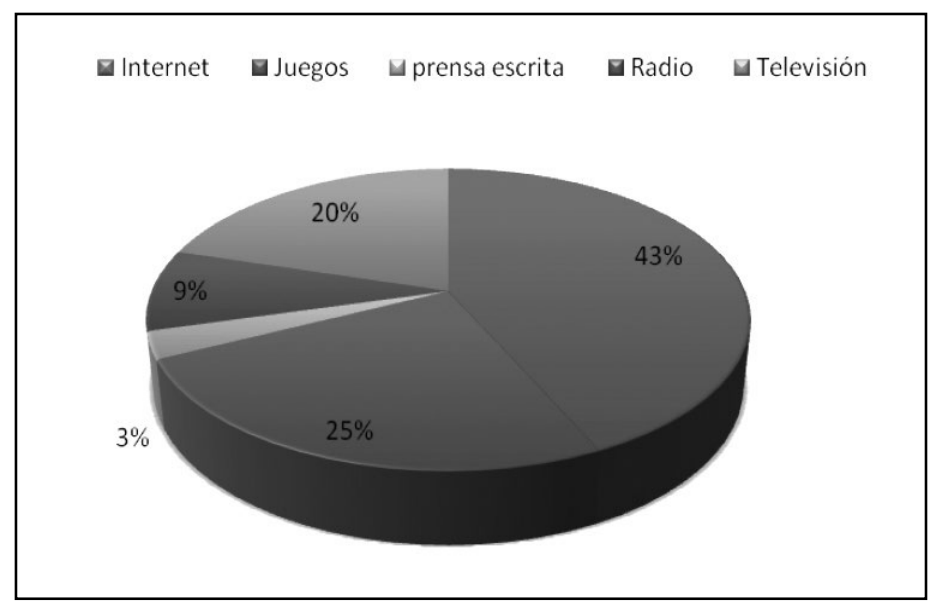

Fuente: Elaboración propia.

\subsection{Tiempo de exposición versus actitud hacia la marca}

La figura 4 muestra que a más horas de exposición al juego, mayor es el cambio de actitud del target hacia la marca. Aquellos que han jugado por más de 3 horas afirman que se sienten más conectados con ella y también representan el grupo que mayor ha consumido la marca después del juego. De la misma manera, aquellos que no han cambiado su actitud hacia la marca son mayormente los que juegan 1 hora. 
Figura 4: Tiempo de exposición versus actitud hacia la marca.

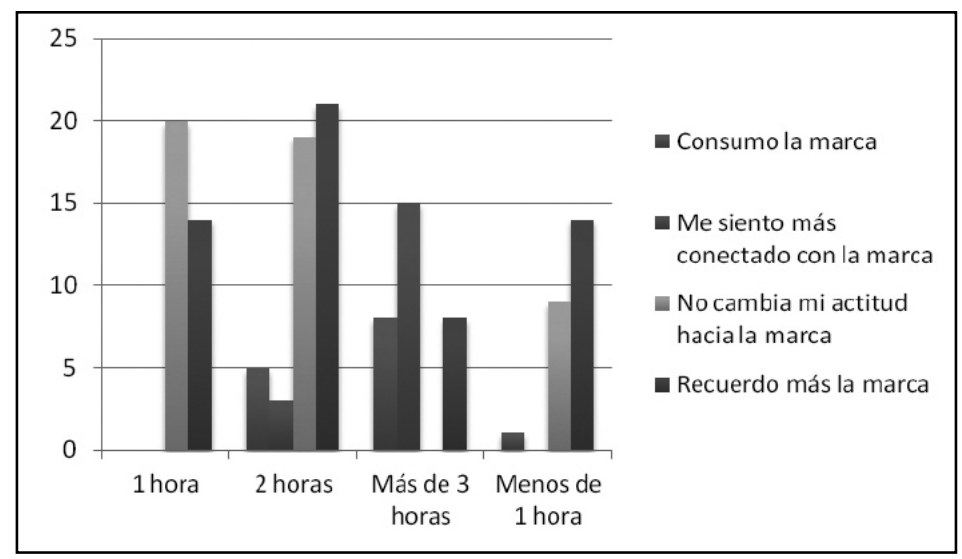

Fuente: Elaboración propia.

\subsection{Frecuencia de uso versus actitud hacia la marca}

La figura 5 indica que a mayor frecuencia de uso por semana, mayor es el cambio de actitud hacia la marca. Es decir, aquellos que han jugado todos los días representan al grupo que ha consumido la marca luego de haber jugado el advergame y aquellos que han jugado como mínimo 3 veces por semana son los que manifiestan sentirse más conectados con la marca. Asimismo, el mayor porcentaje de los encuestados que no han presentado cambios de actitud son aquellos que juegan sólo esporádicamente; lo cual demuestra que la frecuencia de uso impacta a favor del cambio de actitud.

Figura 5: Frecuencia de uso versus actitud hacia la marca.

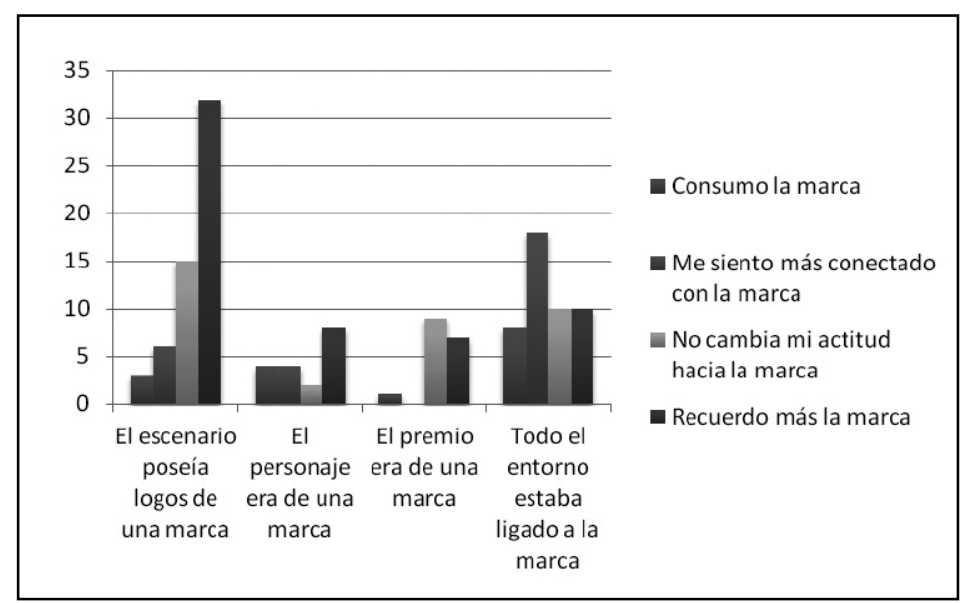

Fuente: Elaboración propia. 


\subsection{Tratamiento de la marca versus actitud hacia la marca}

La figura 6 nos muestra que a mayor presencia de la marca en el juego, mayor es el cambio de actitud hacia ella. Aquellos que han jugado advergames (en los que todo el entorno estaba ligado a la marca) son los que más conexión han hecho con ella y los que la consumen más; a diferencia de aquellos que han jugado advergames con logos insertados en el escenario, cuyo cambio de actitud es únicamente aumentar la recordación de marca en el mejor de los casos.

Figura 6: Frecuencia de uso versus actitud hacia la marca.

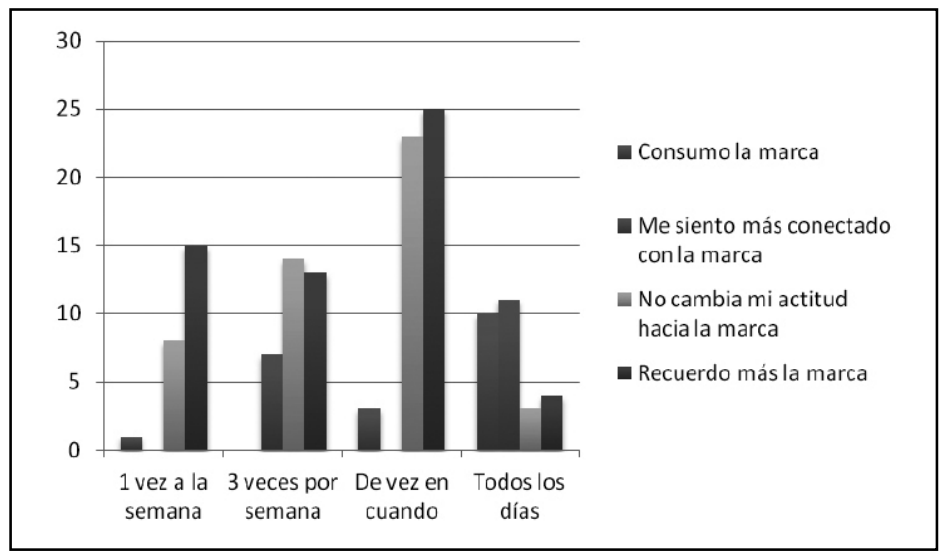

Fuente: Elaboración propia.

\section{Conclusiones}

- Para los alumnos de pregrado de la Universidad de Lima, los advergames son una de las mejores herramientas para hacer publicidad que existe en el mercado actualmente, solamente superada por la Internet, que abarca desde los mismos advergames hasta otras herramientas publicitarias interactivas. Esto quiere decir que los sujetos de estudio prefieren la publicidad que vincula a los usuarios con sus marcas de formas no intrusivas y que les brinda el valor adicional del entretenimiento a cambio de su atención.

- Para lograr un verdadero cambio de actitud hacia la marca, los alumnos de pregrado de la Universidad de Lima manifiestan que los advergames deben configurarse como juegos que vinculen la marca con toda la trama y entorno de la misma y no ser únicamente un medio de emplazamiento de producto o publicidad in-game ya que si bien aumentan la recordación de la marca no generan un cambio de actitud que vincule compra.

- Los estudiantes de sexo masculino son aquellos que poseen la más alta frecuencia de uso y tiempo de exposición a los advergames, ocasionando que sean ellos 
los que logran un vínculo más efectivo con la marca y que en muchos casos se traduce en consumo. Esto quiere decir que los varones es el segmento que mejor puede reaccionar ante esta herramienta para fines publicitarios.

- El tipo de producto más recordado (como protagonista de un advergame) y el que mejor se adapta a los alumnos de pregrado de la Universidad de Lima son las bebidas gaseosas y los snacks, productos consumidos por el público juvenil y que se asocian fácilmente al entretenimiento.

\section{Fuentes consultadas}

América Economía, revista (2013). "Ranking 2013 Perú. Las mejores universidades". Extraída el 17/II/2014 desde http://rankings.americaeconomia.com/mejores-universidadesperu-2013/ranking/

Balseiro, P. (2005). "El marketing boca a boca”. Extraída el 18/II/2014 desde http://www. gestio polis.com/canales $5 / \mathrm{mkt} /$ bocaboca.htm

Bringué Sala, X. y Sádaba Chalezquer, R. (2007). La generación interactiva en Latinoamérica: niños y adolescentes ante las pantallas. Madrid: Fundación Telefónica.

Carcelén García, S. y Sebastián Morillas, A. (2010). “Comunicación viral y buzz marketing”, pp. 107-132. En Sánchez Herrera, J. y Pintado Blanco, J. (coords.) Nuevas tendencias en comunicación. Madrid: Esic Editorial.

Carrera, P. (2008) Teoría de la Comunicación Mediática. Valencia: Tirant lo Blanch.

Dardis, F.; Schmierbach, M. y Limperos, A. (2012). "The Impact of Game Customization and Control Mechanisms on Recall of Integral and Peripheral Brand Placements in Videogames". Journal of Interactive Advertising. Vol. 12, núm. 2, pp. 1-12. Extraída el 18/II/2014 desde http://jiad.org/download87e6.pdf?p=154

Diccionario panhispánico de dudas (2005). "Zapeo". Madrid: Real Academia Española. Extraída el 17/II/2014 desde http://lema.rae.es/dpd/?key=zapping

Eguizábal, R. (2008). Teoría general de la publicidad. Madrid: Eresma \& Celeste.

Guirín, P. (2009, octubre 22). “Advergaming: jugando con las marcas”. Revista Facultades, núm. 38. Extraída el 18/II/2014 desde http://revistafacultades.com.ar/notas/257advergaming-jugando-con-las-marcas.htm

Instituto Nacional de Estadística e Informática (2013). "Las tecnologías de información y comunicación en los hogares. Trimestre: abril-mayo-junio 2013 (en comparación a similar trimestre del año 2011)". Extraída el 18/II/2014 desde http://www.inei.gob.pe/ media/ MenuRecursivo/boletines/17217.pdf 
Ipsos APOYO Opinión y Mercado (2011). “Usos y actitudes hacia Internet 2011”. Extraída el 18/II/2014 desde http://www.ipsos-apoyo.com.pe/sites/default/files/marketing_data/ MKT_data_\%20Internet_2011.pdf

Jiménez, I. (2012, julio 18). "La Publicidad online es una apuesta segura para marcas y anunciantes". Extraída el 18/II/2014 desde http://www.puromarketing.com/ 25/13655/ publicidad-online-apuesta-segura-para-marcas-anunciantes.html

Klein, N. (2003). No logo: el poder de las marcas. Barcelona: Paidós.

Lamb, C.; Hair, J. y McDaniel, C. (2006). Fundamentos de Marketing. México D. F.: Thomson.

Marquès Graells, P. (2001). "Los videojuegos”. Extraída el 18/II/2014 desde http:// peremarques.pangea.org/videojue.htm\#claves

Marti Parreño, J. (2005). Publicidad y entretenimiento en la web. Barcelona: Ra-Ma.

Martín Cabello, A. (2008). “Comunicación, cultura e ideología en la obra de Stuart Hall”. Revista Internacional de Sociología, vol. LXVI, núm. 50, pp. 35-63.

Medina, A. (2007, febrero 21). "La publicidad que viene: advertainment y advergaming". Extraída el 18/II/2014 desde http://www.marketingdirecto.com/punto-de-vista/la-columna/ la-publicidad-que-viene-advertainment-y-advergaming-agustin-medina/

Mencía, J. (2012, agosto 31). "Videojuegos para vender más”. Extraída el 18/II/2014 desde http://www.contunegocio.es/estrategia/videojuegos-para-vender-mas/

Morgado, M. (2008). "Del product placement no se escapa nadie". Extraída el 18/II/2014 desde http://www.uji.es/bin/publ/edicions/jfi13/36.pdf

Muñoz Gallego, A. (2007). “Advergaming 2.0, una nueva forma de hacer publicidad jugando". Extraída el 18/II/2014 desde http://news.omexpo.com/2007/11/advergaming-20-una-nueva-forma-de-hacer-publicidad-jugando-272/

\section{Pons Alfonso, J.}

_(2006a, junio 5). “Advergaming, cuestiones básicas”. Extraída el 17/II/2014 desde http:// www.exelweiss.com/blog/37/advergaming-cuestiones-basicas/

_(2006b, junio 9) “Cinco razones por las que elegir el advergaming” Extraída el 18/II/2014 desde http://www.exelweiss.com/blog/38/5-razones-por-las-que-elegir-el-advergaming/

Severin, J. y Tankard, J. (1979). Communication theories: Origins, Methods, Uses. Nueva York: Hastings House. 